\title{
Discovering and Understanding Android Sensor Usage Behaviors with Data Flow Analysis
}

\author{
Xing Liu • Jiqiang Liu - Wei Wang . \\ Xiangliang Zhang
}

the date of receipt and acceptance should be inserted later

\begin{abstract}
Today's Android-powered smartphones have various embedded sensors that measure the acceleration, orientation, light and other environmental conditions. Many functions in the third-party applications (apps) need to use these sensors. However, embedded sensors may lead to security issues, as the third-party apps can read data from these sensors without claiming any permissions. It has been proven that embedded sensors can be exploited by well designed malicious apps, resulting in leaking users' privacy. In this work, we are motivated to provide an overview of sensor usage patterns in current apps by investigating what, why and how embedded sensors are used in the apps collected from both a Chinese app market and official market called Google Play. To fulfill this goal, We develop a tool called "SDFDroid" to identify the used sensors' types and to generate the sensor data propagation graphs in each app. We then cluster the apps to find out their sensor usage patterns based on their sensor data propagation graphs. We apply our method on 22,010 apps collected from AppChina and 7,601 apps from Google Play. Extensive experiments are conducted and the experimental results show that most apps implement their sensor related functions by using the thirdparty libraries. We further study the sensor usage behaviors in the third-party libraries. Our results show that the accelerometer is the most frequently used sensor. Though many third-party libraries use no more than four types of sensors, there are still some third-party libraries registering all the types of sensors recklessly. These results call for more attentions on better regulating the sensor usage in Android apps.
\end{abstract}

Keywords Android system; Sensor usage; Data-flow analysis; Clustering

Corresponding author: Wei Wang

Xing Liu · Jiqiang Liu . Wei Wang

School of Computer and Information Technology, Beijing Jiaotong University, 3 Shangyuancun, 100044 Beijing, China

E-mail: xingliu, wangwei1, jqliu@bjtu.edu.cn

Xiangliang Zhang

Division of Computer, Electrical and Mathematical Sciences \& Engineering,

King Abdullah University of Science and Technology, Saudi Arabia

E-mail: xiangliang.zhang@kaust.edu.sa 


\section{Introduction}

Android-powered smartphones have become more and more popular for both personal and business use. According to a report from the International Data Corporation (IDC) [18], Android-powered smartphones dominate the market with a $87.6 \%$ share in the second quarter of 2016. At the same time, the smartphone's hardware is more and more advanced. Various sensors have been embedded in smartphones, such as the motion sensor (e.g., accelerometers, gravity sensors, gyroscopes, rotational vector sensors), the environmental sensor (e.g., temperature, illumination) and the position sensor (e.g., orientation sensors and magnetometers) [1]. These sensors are used by some third-party applications (apps) to support their novel features, such as the Spirit Level in some Camera-related apps.

Android is designed without considering the security issues that may be led by the embedded sensors. The third-party apps can use embedded sensors without claiming any permissions on Android platform. However, embedded sensors can be exploited by well designed malicious apps (malapps), resulting in leaking users' privacy. For example, tapping different positions on the touchscreen will cause different motion changes of the smartphone. If the correlations between the tap events and the data collected by the motion sensors are learned, one can successfully guess users' input through the data collected by the motion sensors. Related work [7, $8,25,28,30]$ has already demonstrated that smartphones' embedded sensors can expose users' privacy data. In particular, Xu et al. [36] designed and implemented "TapLogger", a trojan app that uses obtained sensor data to log user inputs on touchscreen stealthily. "TapLogger" was reported by various media $[26,32]$ due to its high threat to users' confidential information. Besides, other researchers $[20$, $22,40]$ proposed many user identity recognition mechanisms based on embedded sensor data. Their work indicates that embedded sensors' data can not only be used to identify whether a user is the smartphone's owner, but can also leak users' identity information.

Although these studies still remain in the experimental stage, they attract us to discover the sensor usage patterns in the current Android apps. According to a statistical result from AppBrain [5], the number of apps in Google Play has reached 2.4 million, while according to the Android API Guides [1], Android system supports more than ten types of sensors. Since embedded sensors can expose confidential information on smartphones, curiosities are aroused on understanding what, why and how the embedded sensors are used in current apps. Previous work does not answer this questions, since the related work usually focuses on Android permission analysis [33], Android malware detection [31] or user privacy protection in Android platform [24]. In order to better answer these questions, we extend our previous work [23] by additionally analyzing 7,601 popular apps from Google Play and discuss the experimental results in depth. We design and implement a tool called SDFDroid (Sensor Data Flow Droid). It first disassembles the apk files to smali code files, and then performs two kinds of data flow analysis. One is backward tracking analysis, which starts from the API that registers a sensor listener in the system to find the used sensors' types. The other is forward tracking analysis, which starts from the API that reads sensor data from the system to generate sensor data propagation graphs. We calculate the similarity between each pair of sensor data propagation graphs that are generated from many apps, and then cluster the graphs with DBSCAN [13], a well known clustering algorithm. Sensor 
usage patterns are thus constructed from the clustering results. We analyze 22,010 apps collected from AppChina (one of the main Android app markets in China) and 7,601 apps from Google Play. We find that almost all the apps implement sensor related functions with the help of the third-party libraries. We also find that accelerometer is the most-used sensor and that the used sensor type has a clear association with the app's function. We make the following contributions in this paper:

- We design and implement SDFDroid that fast and accurately analyzes Android apps to identify the types of embedded sensors used by the apps and to generate the apps' sensor data propagation graphs.

- We employ SDFDroid to analyze all the apps in AppChina and some popular apps in Google Play. We reveal sensor usage patterns in apps at a large scale.

- To the best of our knowledge, our work is the first attempt to discovering the sensor usage behaviors on the view of a complete Android app market.

The remainder of this paper is organized as follows. Section 2 describes the background of Android sensor system and smali code files. Section 3 gives our system design. Section 4 presents experimental results and limitations. Related work is given in Section 5. Section 6 concludes our work.

\section{Background}

\subsection{Android Sensor System}

Today's Android-powered smartphones are equipped with various embedded sensors. These sensors are used to monitor the device's movement, position or other surrounding environmental conditions. The Android system supports many types of sensors (see Table 1). Some sensors are hardware types, which means that the sensor data is directly read from physical sensors built in the smartphone. Other sensors are software types, which means that the sensor data is read and calculated from one or more of the hardware sensors [1]. The embedded sensors are widely used in the third-party apps. For example, a navigation app may use the magnetic field sensor to determine the direction.

The embedded sensors are managed by Android Sensor Framework. Different from the Camera, GPS and Bluetooth which are protected by Android permission mechanism, the embedded sensors can be directly used by the third-party apps without any requirement of permissions. With the help of Android Sensor Framework, a third-party app can read a sensor's data by the following steps (as shown in Figure 1).

- First, instantiate an object of SensorManager class (Line 12). In this step, an app creates an instance of the sensor service. This class provides many methods for using sensors, such as accessing specified sensors and listing all available sensors, registering and unregistering sensor event listeners.

- Second, instantiate an object of Sensor class by calling the getDefaultSensor() (Line 14). In this step, an app gets an object of Sensor with a specific type. The type of the sensor can be specified by the method's parameter. As shown in this example, Sensor.TYPE_ACCELEROMETER is a constant describing an 
Table 1 Some sensor types supported by the Android platform

\begin{tabular}{|l|l|l|}
\hline Sensor & Type & Description \\
\hline Accelerometer & Hardware & Measures the acceleration force \\
\hline Ambient_Temperature & Hardware & Measures the ambient room temperature \\
\hline Gravity & Software or Hardware & Measure the force of gravity \\
\hline Gyroscope & Hardware & Measures a device's rate of rotation \\
\hline Light & Hardware & Measures the ambient light level \\
\hline Linear_Accelerometer & Software or Hardware & $\begin{array}{l}\text { Measures the acceleration force, } \\
\text { excluding the force of gravity }\end{array}$ \\
\hline Magnetic_Field & Hardware & Measures the ambient geomagnetic field \\
\hline Orientation & Software & $\begin{array}{l}\text { Measures degrees of rotation that a device } \\
\text { makes around all three physical axes }\end{array}$ \\
\hline Pressure & Hardware & Measures the ambient air pressure \\
\hline Proximity & Hardware & $\begin{array}{l}\text { Measures the proximity of an object } \\
\text { relative to the view screen of a device }\end{array}$ \\
\hline Relative_Humidity & Hardware & Measures the relative ambient humidity \\
\hline Rotation_Vector & Software or Hardware & Measures the orientation of a device \\
\hline Temperature & Hardware & Measures the temperature of the device \\
\hline
\end{tabular}

$1 / /$ SensorActivity.java

2 public class SensorActivity extends Activity

3 \{

4 float accx;

5 private SensorManager sensorManager:

6 private Sensor sensora;

8 public void onCreate(Bundle paramBundle)

92

10 super.onCreate(paramBundle);

11 setContentView(R.mainview);

12 sensorManager = (SensorManager) getSystemService (Context.SENSOR_SERVICE)

I3 Log.d("sensor", "Get sensorManager Success");

14 sensora = sensorManager. getDefaultSensor(Sensor.TYPE_ACCELEROMETER);

15 SensorEventListener accelerometerListener = new SensorEventListener()

16\{

17 public void onAccuracyChanged(Sensor arg0, int arg1)

18 \{

20

21

22

23

24
25
26

26

$\left.\begin{array}{l}27 \\ 28 \\ 29\end{array}\right\}$

$29 \quad \ldots$.

, 30$\}$

pubic

public void onSensorChanged(SensorEvent $\arg 0$ )

$\operatorname{accx}=\arg 0$. values $[0]$

.......

// Do something with this sensor value

;

this.sensorManager.registerListener( accelerometerListener, sensora, SENSOR_DELAY_NORMAL);

Fig. 1 Code example to use sensor

accelerometer sensor type. If light sensor is needed, one can set the method's parameter as Sensor.TYPE_LIGHT. All available constants can be found in the Android developer website [2].

- Third, instantiate an object of SensorEventListener interface (Line 15) and override two callback methods which are onAccuracyChanged() (Line 17) and onSensorChanged() (Line 20). These two methods are used to receive sensor events when sensor accuracy changes or when sensor values change. Android 
system will invoke onSensorChanged() when sensor data changes automatically, and a SensorEvent object is put in the methods' parameter. The SensorEvent object is created by the system. It contains the following information: the raw sensor data, the sensor's type, the accuracy of the data and the timestamp of this event. Developers can read sensor data from this object (Line 22).

- Last, register a sensor event listener (Line 27). By invoking the method registerListener(), app registers a SensorEventListener into the system. The sensor that will be registered is specified by the method's second parameter. When a sensor's value changes, the system will only notify the apps that have registered this sensor. The third parameter of this method is used to set the data delay. The data delay controls the interval at which sensor events are sent to the app. In this example, the default data delay (SENSOR_DELAY_NORMAL) let this app receive the raw sensor values every 0.2 seconds.

\subsection{Smali Code}

Smali code is a kind of programming language used by Dalvik, Android's JVM implementation. A given app's smali codes can be retrieved with Apktool [4], a tool for reversing Android apps. An app's smali codes obtained by disassembling are matched with the app's source codes. Hence, analyzing the smali codes directly is reasonable. We provide the corresponding disassembled smali codes of Figure 1 in Figure 2 and Figure 3. Figure 3 is the smali codes associated with the interface class SensorEventListener. Apktool generates individual smali files for interface classes and inner classes. In the smali files, all instructions are based on registers. Local registers are represented with the symbols start with $v$, parameter registers are represented with the symbols start with $p$. Operations on the registers are determined by the Opcodes, such as $\{$ const-string, invoke-static, iput-object, ... $\}$. For example, const-string v2, "sensor" (in Figure 2 Line 16) means creating a new string whose value is "sensor" and putting it in register $v 2$. Detailed information about the meanings of different Opcodes can be found in [3].

\section{System Design}

\subsection{High-level Overview}

We design and implement a tool called "SDFDroid" that performs static analysis on Android apps to identify the apps' used sensor types and to generate the apps' sensor data propagation graphs. SDFDroid supports automated analysis and is efficient to process a large number of apps. After we get all the apps' sensor data propagation graphs, we calculate their similarity. We convert the similarity into distance between each pair of graphs before we cluster the graphs. In this work, DBSCAN [13] is employed to cluster the app samples based on the distances between each pair of graphs, as DBSCAN is a widely-used clustering algorithm based on density estimation that can describe the behaviors of various apps. The sensor usage patterns of each cluster are then analyzed based on the clustering results. We illustrate the system's overview in Figure 4. 


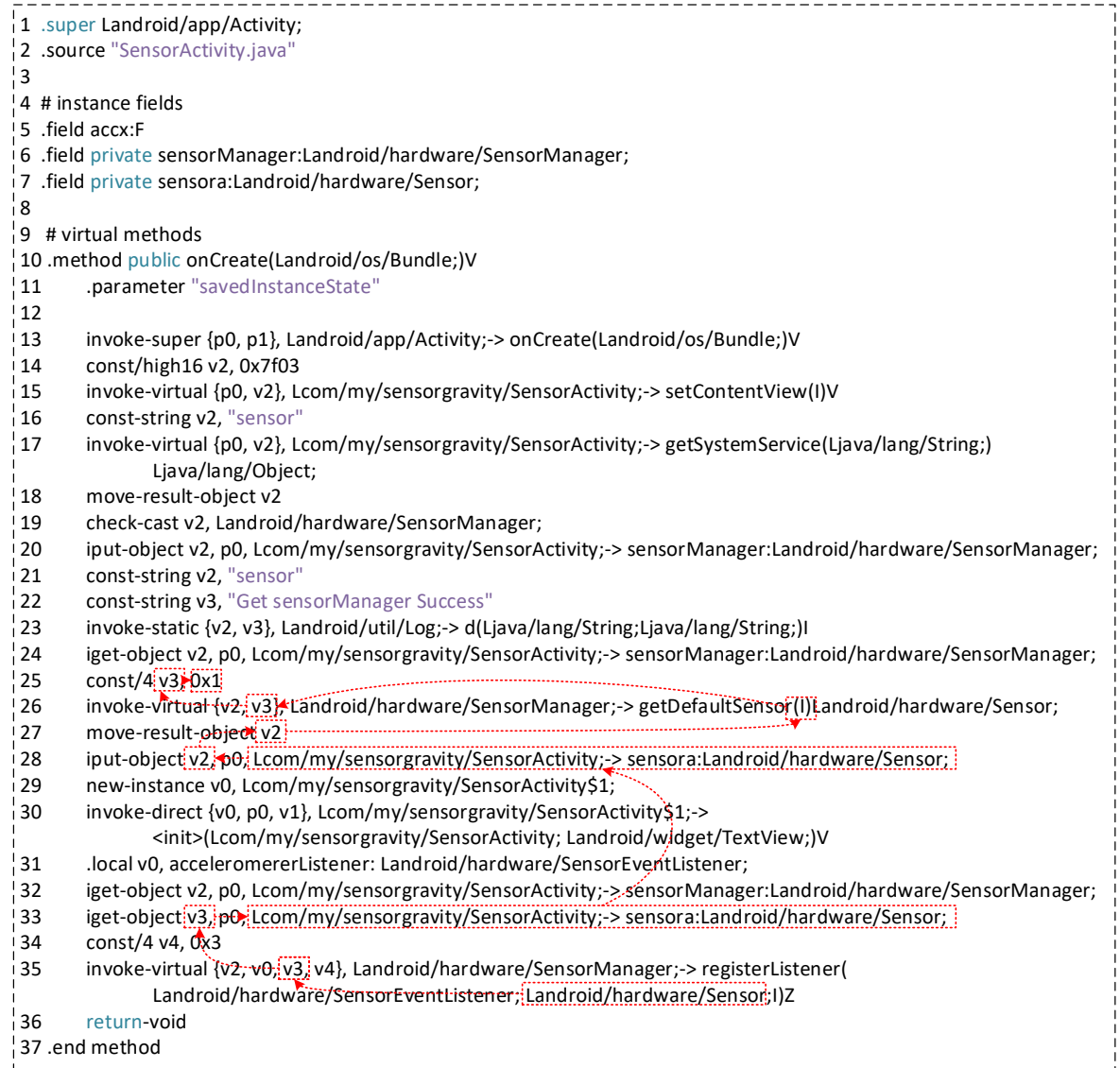

Fig. 2 Smali code for SensorActivity

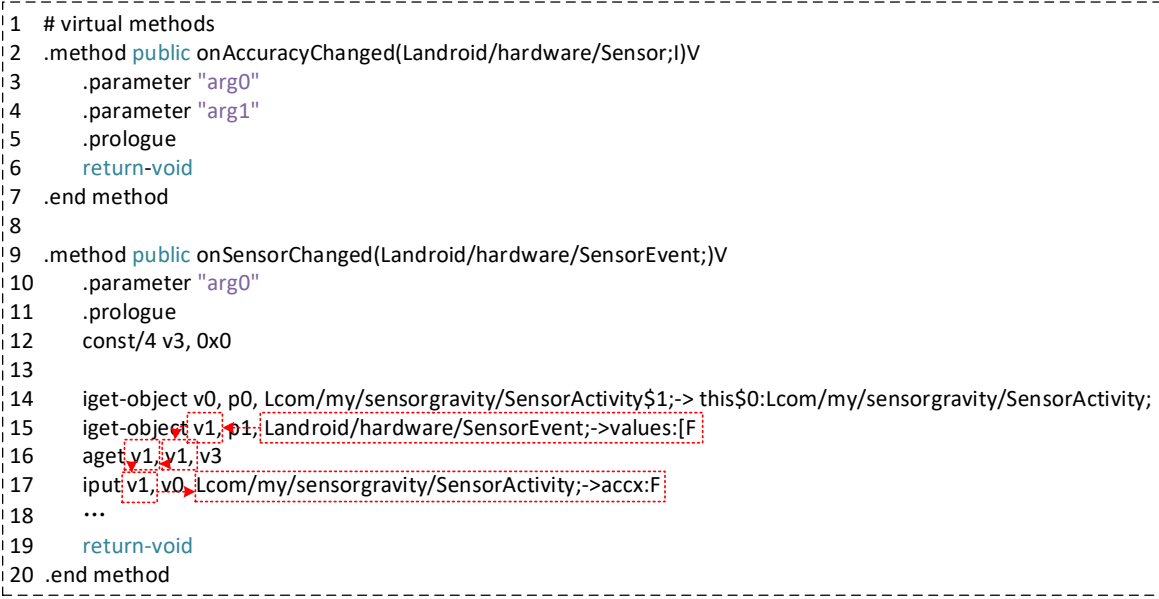

Fig. 3 Smali code for SensorActivity $\$ 1$ 


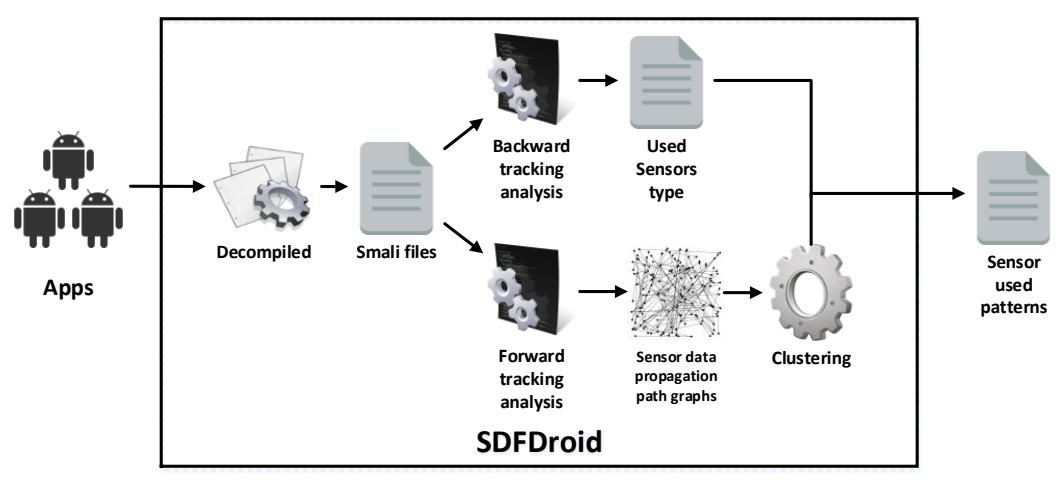

Fig. 4 Overview of SDFDroid

\subsection{Static Analysis}

In this Section, we describe the static analysis method used in this work. Complete code is statically analyzed without the need of its execution. Therefore, static analysis can be done at app market level efficiently.

An Android app is packaged as an Android application package (APK) file with compiled bytecode, additional metadata and resources in it. SDFDroid disassembles an APK file into smali code files with the help of Apktool. Then, SDFDroid parses the smali files and creates corresponding java objects of their contents.

Based on the previous step, SDFDroid performs two kinds of static analysis. One is backward tracking analysis that specifically focuses on analyzing registerListener()'s second parameter to find out which sensors are used by an app. The other is forward tracking analysis that focuses on generating an app's sensor data propagation graph. We describe the detail of these two kinds of static analysis as follows (we use the codes in Figure 2 and Figure 3 for examples):

- Backward tracking analysis. In the backward tracking analysis, the analysis begins at the method registerListener(), whose second parameter specifies the used sensor. SDFDroid first finds out the corresponding smali code of registerListener() in the smali code files, then begins to track the register $v 3$ that stores the value of this method's second parameter (see line 35, Figure 2). SDFDroid searches the smali codes in reverse order to find the value of $v 3$. Then, SDFDroid finds that $v 3$ obtains an object value at line 33 . This object is a class field defined in Line 7. In the next step, SDFDroid searches the corresponding setters of this field. One setter can be found in Line 28. The value in register $v 2$ is assigned to this field. After this, SDFDroid backtracks register $v 2$, and finds that $v 2$ stores the result of invoking method getDefaultSensor (see Line 26-27). This method is provided by Android system, and we know the return value of this method is decided by the method's parameter. Hence, the register corresponding to this method's parameter is backtracked (See register $v 3$ in Line 26). If a register obtains a constant value, the tracking of this register will be terminated, and the tracking result will be reported. As shown in this example, register $v 3$ gets a constant value in Line 25 . This result will be reported, and we will realize the sensor type is 1 . Hence, the sensor used by this 
app is an accelerometer. The relationships between the numbers and the real sensor types are listed in Android Developer Website [2].

- Forward tracking analysis. The forward tracking analysis is similar with the backward tracking analysis. SDFDroid first creates an empty graph and finds out the register that needs to be tracked in the smali codes. Then, it determines whether the value of this register is assigned to another object, or is overwritten by another value. In the forward tracking analysis, if the value of a tracked register is assigned to another object, the target object that receives the value is added into the track queue. Otherwise, if the register is overwritten, the tracking of this register will be stopped. For example, in Figure 3, sensor data is obtained from the system through the SensorEvent object: Landroid/hardware/SensorEvent;->values:[F and is assigned to the register $v 1$ (See Line 15). SDFDroid searches the smali files in order in forward tracking analysis. Finally, SFDroid finds the value of register $v 1$ is assigned to a class field accx in Line 17. At the same time, the search process reaches the end of this method. Hence, the tracking of register $v 1$ is stopped, and SDFDroid will search at where the class field $a c c x$ is used in all other smali code files of the same app in the next step. Each time SDFDroid finds a new line of smali code that propagates the sensor data, it adds the smali code into the graph as a node. The directions of sensor data propagation are represented by the directed edges between each pair of nodes.

Both backward tracking analysis and forward tracking analysis have a queue to store objects that need to be tracked. Once the tracking process finds an object that needs to be tracked, it puts the object in this queue. When the tracking of the current object is finished, SDFDroid gets an object from the top of the queue and starts to track it. The static analysis of an app is finished when the queue is empty. An app's used sensor types are reported in a .xml file while its sensor data propagation graph is reported in a .gexf file. GEXF(Graph Exchange XML Format) is a language for describing complex network structures. Gephi[15] can open a .gexf file and show the graph (See Figure 5). Nodes in the graph are smali code lines, while edges represent data propagation directions.

\subsection{Clustering Analysis}

Once the used sensor types and sensor data propagation graphs of all the apps are generated, we perform clustering analysis on these graphs to construct their sensor usage patterns.

\subsubsection{Distance calculation}

The first step to perform clustering analysis is to calculate the similarity between each pair of graphs. Calculating the edit distance between two graphs is an effective way of calculating the similarity between these two graphs. Graph edit distance measures the minimum number of graph edit operations (including insert or delete a node, insert or delete an edge or change the label of a node or edge) to transform one graph to another. However, calculating the edit distance between two graphs is NP-hard [37]. It is only applicable to graphs that have a small number of nodes. 


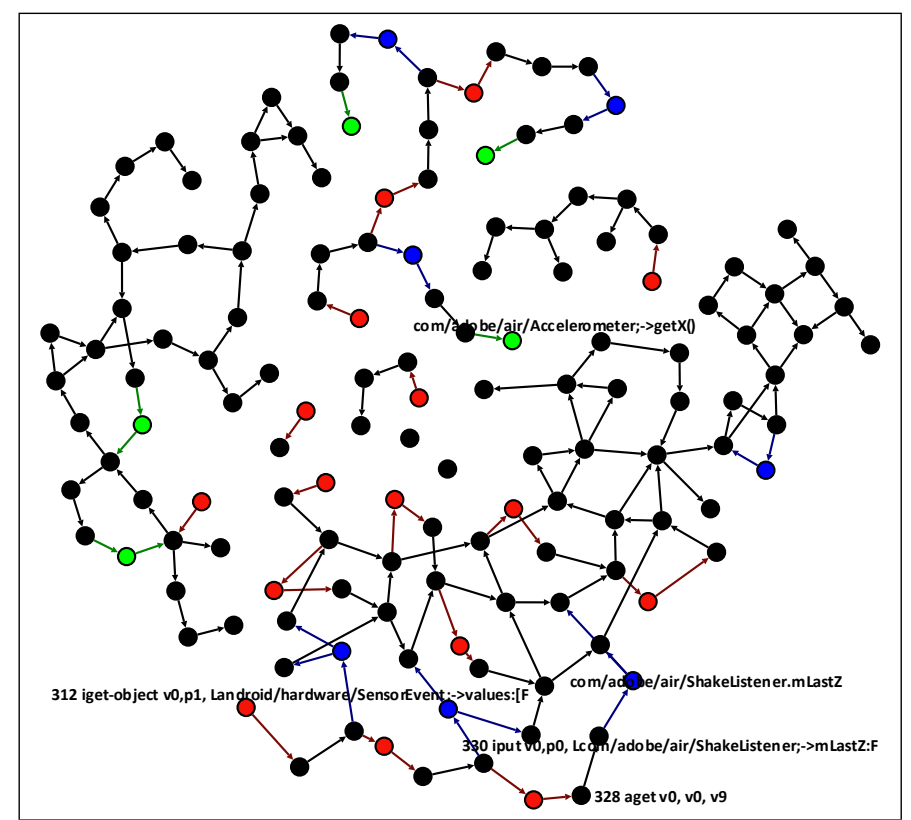

Fig. 5 Sensor Data Propagation Path Graph.

Although many researchers have modified the basic algorithm of graph edit distance to improve its efficiency, it cannot be applied to the sensor data propagation graphs that may have hundreds of nodes. Besides, an Android app market may have more than ten thousand apps that need to be analyzed. Since we need a fast algorithm to calculate the similarity between each pair of graphs on a large-scale, in this work, we compute a graph hash for each graph and calculate the similarity between each pair of graphs based on their hash values. This procedure is inspired by Neighborhood Hash Graph Kernel (NHGK) that is originally proposed by Hido and Kashima [16].

NHGK is a kernel operation over labeled graphs. It has high expressiveness of the graph structure, and fast execution speed on graphs with large number of nodes. Hence, it is applicable to process graphs with hundreds of nodes such as the sensor data propagation graphs. The main idea of NHGK is to integrate the information of a node and its neighbor nodes into a hash value. The calculation of a node's hash value is defined as follows:

$$
H(v)=R(l(v)) \oplus\left(l\left(v_{1}^{N E}\right) \oplus, \cdots, \oplus l\left(v_{n}^{N E}\right)\right)
$$

where $l(v)$ represents the binary label that is transformed from the node's real label. $\oplus$ represents a bit-wise XOR. $R$ means a one-bit rotation to the left. $v^{N E}$ is the neighbor nodes of node $v$. This algorithm can also be used $k$ times iteratively to integrate information across neighbors up to a path with length $k$. The hash value of a graph $G$ is obtained by calculating the hash values of all the nodes in graph $G$. Then the similarity between two graphs is computed based on the number of nodes that have the same hash values (Algorithm 1). In this algorithm, the binary hash values are treated as integer. Hence, they can be sorted and compared. 


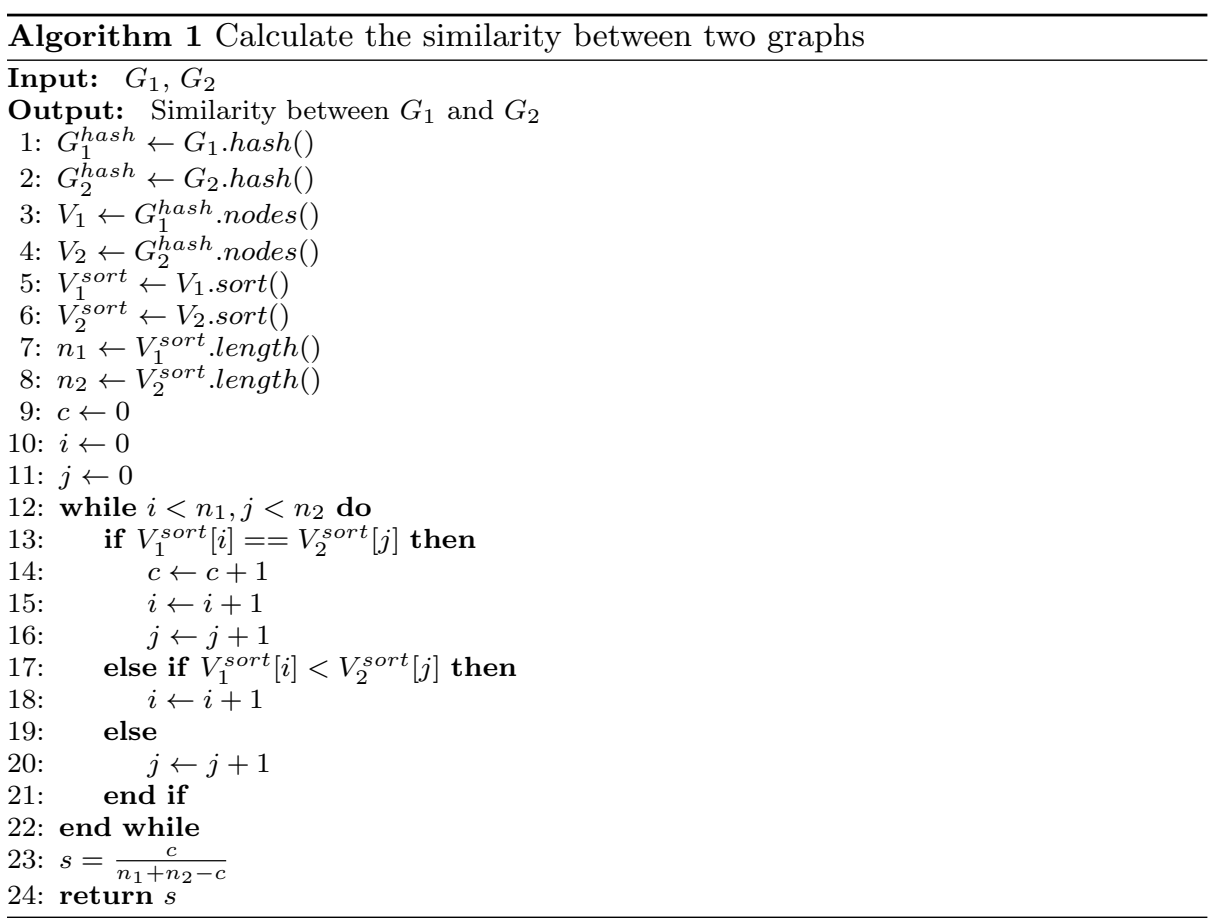

In our work, when we calculate the similarity between each pair of graphs, we simplify the labels of nodes in the graphs at first to ensure the method's robustness. The labels of nodes are entire smali code lines in original graphs (as shown in Figure 5), and we relabel the nodes only with the operation codes. For example, node label mul-float v1,v2,v3 is relabeled as mul-float. For some special operations, such as invoking a method, we also reserve the names of the invoked methods. Then we count the number of distinct labels in all graphs, and relabel them with different binary labels. Finally, each node's hash value is computed. By doing this, we can calculate the similarity between each pair of sensor data propagation graphs with the algorithm above.

\subsubsection{Clustering}

After we have calculated the similarity between each pair of graphs, we cluster the graphs to find their common characteristics and thus to discover the sensor usage patterns of different apps. The algorithm we used is Density-based spatial clustering of applications with noise (DBSCAN) [13]. DBSCAN is one of the most widely-used clustering algorithms based on density. The main idea behind this algorithm is that, for a set of points in a given space, it clusters points that are closely located together and make outliers for points that lie alone in low-density regions. In a DBSCAN clustering, the points are classified as core points, reachable points and noise. A core point $p$ is a point that has at least $m$ neighbor points within distance $d$ from it, and these neighbor points are considered to be directly reachable from $p$. A point $q$ is a reachable point of $p$ if there is a path from $p$ to $q$ 

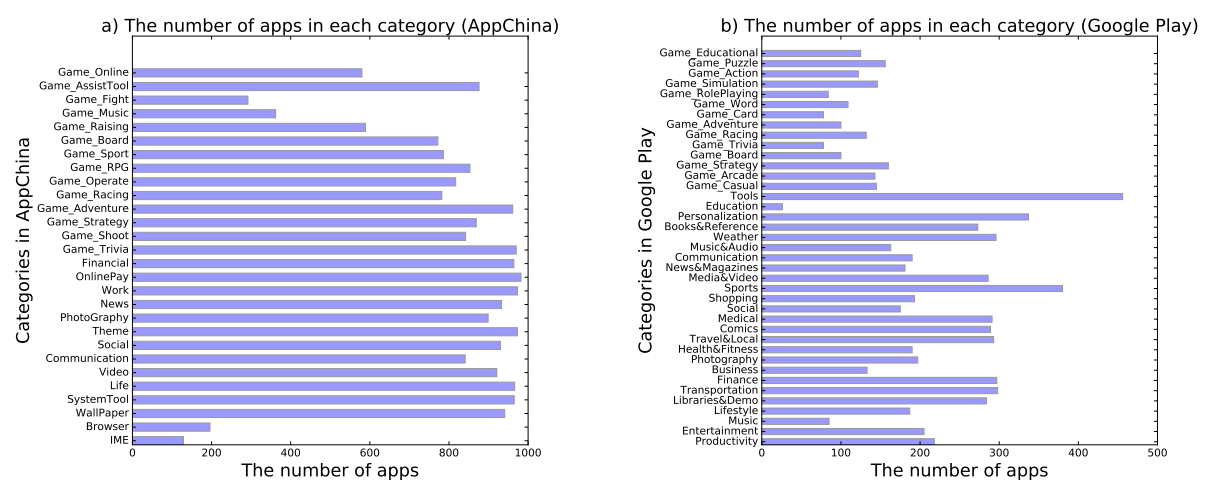

Fig. 6 The number of apps in each category

where each point on the path can be directly reached from the point before it. The points are neither core points nor reachable points are noise. After the clustering, each cluster consists of some core points and their reachable points. The points that are reachable but not core points form the cluster's edge [35].

DBSCAN only needs two parameters: $m$ and $d$ to confirm core points. It does not need to specify the number of clusters. Therefore, it is suitable on our app set, as we do not know how many clusters the apps will be associated with.

In our work, we transform the similarity between each pair of graphs generated with SDFDroid to a distance between each pair of graphs. For two graphs, the more similar they are, the smaller distance they have. We measure the distance by:

$$
d=\frac{1-s}{1+s}
$$

where $s$ is the similarity between two graphs and $d$ is the distance.

\section{Evaluation}

\subsection{Dataset}

The dataset in the experiments is collected from two app markets. One is AppChina, a famous Android app market in China. We download all the 22,010 apps available in AppChina in December 2014. The apps from AppChina belong to 14 subcategories under "Software" category and 14 subcategories under "Game" category. The other one is Google Play. We download 7,601 popular apps available in Google Play in July 2016. The apps from Google Play belong to 25 subcategories under "Apps" category and 14 subcategories under "Games" category. The number of apps in each subcategory is shown in Figure 6. For each app, we get it's apk file, size, developer and description.

\subsection{Analysis Results}

Except some apps that cannot be disassembled by Apktool, we successfully analyzed 19,914 apps from AppChina and 7,601 apps from Google Play. We find 
10,976 apps (55.1\%) from AppChina and 3,179 apps (41.8\%) from Google Play contain code snippets that are used to read sensor data. We present the detailed cluster and analysis results as follows.

\subsubsection{Clustering results}

In order to ensure the robustness and representative, in the process of clustering, we set the parameter $d$ and $m$ of DBSCAN to 0.1 and 10 respectively. Apps from AppChina and apps from Google Play are clustered separately. After the clustering, 8,319 apps from AppChina are clustered into 98 clusters and 1,468 apps from Google Play are clustered into 35 clusters. We compare the samples in the same cluster and find the main reason why their sensor data propagation graphs are similar is the using of the same third-party libraries. Table 2 shows the sensor related third-party libraries used in the AppChina's clusters that have more than 100 apps. Some third-party libraries' methods are obfuscated with different names in different versions, which makes their sensor data propagation graphs different, hence they are clustered into different clusters. The table shows Tencent, Cocos2dx, Unity3d are the Top 3 frequently used third-party development libraries which contain sensor related codes in the apps from AppChina. Com.tencent.mm.sdk is a Jar package used to share the moments with one's friends in WeChat (The most widely-used IM tool in China). Cocos2dx is a framework for building 2D games and other graphical apps. Unity3d is a game development toolkit that helps developers to build 3D games and real time 3D animations. Table 3 shows the sensor related third-party libraries used in the Google Play's clusters that have more than 20 apps. This table displays Unity3d, Tapjoy, Google ad are the most frequently used sensor related third-party libraries in the apps from Google Play. Tapjoy is an advertisement (ad) library. com.google.android.gms.ads is also used for ad purpose. We also manually check the other libraries and find most of them are ad libraries and game development libraries. These results show running games and displaying ads are the main reasons for apps using embedded sensors.

Figure 7 is a matrix describing clustering results by category for apps from AppChina. This figure illustrates that different category usually has different sensor usage patterns. Category IME, Browser and Theme have few apps that have sensor related codes. Most apps in Category Game_Trivia, Game_Shoot, Game_Strategy and Game_Adventure are classified into Cluster 15 and Cluster 16. It indicates that most apps in these categories are developed with the help of Cocos2dx and Unity3d. Each category contains some apps that have sensor related code snippets. Figure 7 also shows some clusters only contain apps in "Software" category while some other clusters only contain apps in "Game" category. This reveals the different sensor usage behaviors between apps and games. This figure also shows "Game" apps are more likely to use sensors. Figure 8 is a matrix characterizing clustering results by category for apps from Google Play. This figure demonstrates that Unity3d and Cocos2dx are usually used in "Game" apps because most apps in cluster 1 and cluster 5 are games. Figure 8 also shows most apps in cluster 6 are "Apps" apps and most apps in cluster 19 are "Game" apps, which indicates that Google ad is usually used in "Apps" apps and Tapjoy is commonly used in "Game" apps. We also study the reason why some clusters contain many apps from the same category. We find the apps in the same combination of cluster and category are usually developed by the same developer based on the same template. For 
Table 2 The sensor related third-party libraries in the clusters from AppChina

\begin{tabular}{|c|c|c|c|}
\hline ID & Cluster Number & The Number Of Apps & Sensor Related Third-Party Libraries \\
\hline 1 & 15 & 1097 & org. $\operatorname{cocos} 2 \mathrm{dx}$.lib \\
\hline 2 & 16 & 938 & com.unity3d.player \\
\hline 3 & 1 & 619 & com.tencent.mm.sdk \\
\hline 4 & 14 & 398 & com.tencent.mm.sdk \\
\hline 5 & 6 & 397 & com.tencent.mm.sdk \\
\hline 6 & 3 & 313 & org. $\operatorname{cocos} 2 \mathrm{dx}$.lib \\
\hline 7 & 25 & 214 & com.adobe.air.Accelerometer \\
\hline 8 & 24 & 196 & com.badlogic.gdx.backends.android \\
\hline 9 & 13 & 185 & org.apache.cordova \\
\hline 10 & 43 & 177 & com.mobi.view \\
\hline 11 & 10 & 166 & $\begin{array}{l}\text { com.tencent.mm.sdk } \\
\text { org.cocos } 2 \mathrm{dx} . \mathrm{lib}\end{array}$ \\
\hline 12 & 34 & 165 & $\begin{array}{l}\text { com.unity3d.player } \\
\text { com.tencent.mm.sdk } \\
\text { com.millennialmedia.android.AccelerometerHelper } \\
\text { com.mobclix.android.sdk }\end{array}$ \\
\hline 13 & 39 & 160 & com.unity3d.player \\
\hline 14 & 62 & 137 & org.andengine.engine.Engine \\
\hline 15 & 40 & 130 & cn.sharesdk.onekeyshare.Shake2Share \\
\hline 16 & 33 & 112 & $\begin{array}{l}\text { com.adsmogo.mriad } \\
\text { com.tapjoy.mraid } \\
\text { org.ormma.controller }\end{array}$ \\
\hline 17 & 9 & 105 & com.adobe.air \\
\hline 18 & 67 & 104 & $\begin{array}{l}\text { com.baidu.location } \\
\text { cn.sharesdk.onekeyshare.Shake2Share }\end{array}$ \\
\hline 19 & 8 & 102 & $\begin{array}{l}\text { com.umeng.socialize.sensor.UMShakeSensor } \\
\text { com.unity3d.player } \\
\text { com.zhuohuamg.game.util.ShakeListener } \\
\text { com.tencent.mm.sdk }\end{array}$ \\
\hline 20 & 27 & 101 & com.trid.tridad.TriDContentsView \\
\hline
\end{tabular}

Table 3 The sensor related third-party libraries in the clusters from Google Play

\begin{tabular}{llll}
\hline ID & Cluster Number & The Number Of Apps & Sensor Related Third-Party Libraries \\
\hline 1 & 1 & 242 & com.unity3d.player \\
2 & 6 & 209 & com.google.android.gms.ads.internal.overlay \\
3 & 5 & 127 & org.cocos2dx.lib \\
4 & 13 & 109 & com.adobe.air \\
5 & 19 & 100 & com.tapjoy.mraid \\
6 & 3 & 60 & com.ansca.corona.CoronaSensorManager \\
7 & 7 & 57 & com.mixpanel.android.viewcrawler.FlipGesture \\
8 & 20 & 53 & com.badlogic.gdx.backends.android \\
9 & 8 & 51 & com.admarvel.android \\
10 & 10 & 49 & com.tapjoy.mraid \\
& & 38 & com.badlogic.gdx.backends.android \\
11 & 9 & 31 & com.facebook.ads \\
12 & 22 & 28 & com.google.android.gms.ads.internal.overlay \\
13 & 30 & 26 & com.ideaworks3d.marmalade.LoaderThread \\
14 & 15 & & com.tapjoy.mraid \\
& & &
\end{tabular}

instance, for cluster 14 and category "WallPaper" in AppChina, 397 apps are developed by the same developer. For cluster 13 and category "Game_Educational" in Google Play, 20 apps are developed by the same developer. Since the developers only need to change some resource files (pictures, text contents, etc.) in the templates, the apps developed with the same template usually have the similar sensor data propagation graphs. 


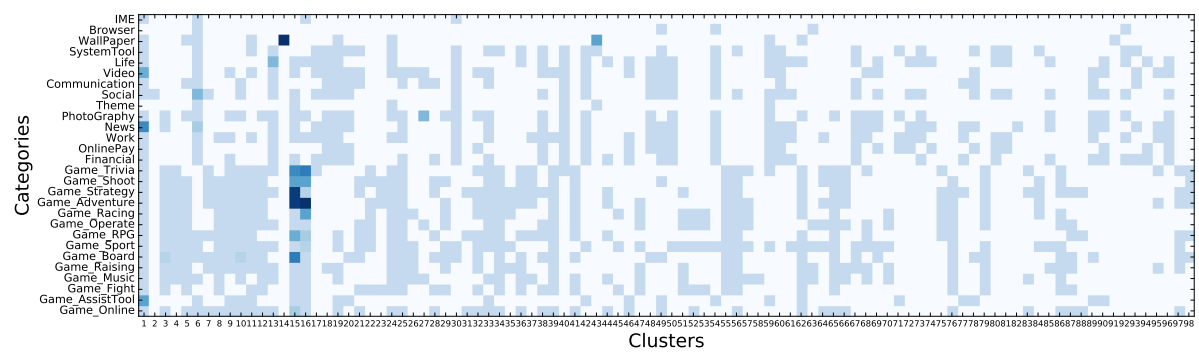

Fig. 7 Matrix for cluster result by category of apps from AppChina. Each grid corresponds to a combination of cluster and category, and the depth of the grid's color represent the number of apps in this grid. The more apps in the corresponding combination of cluster and category, the deeper the color.

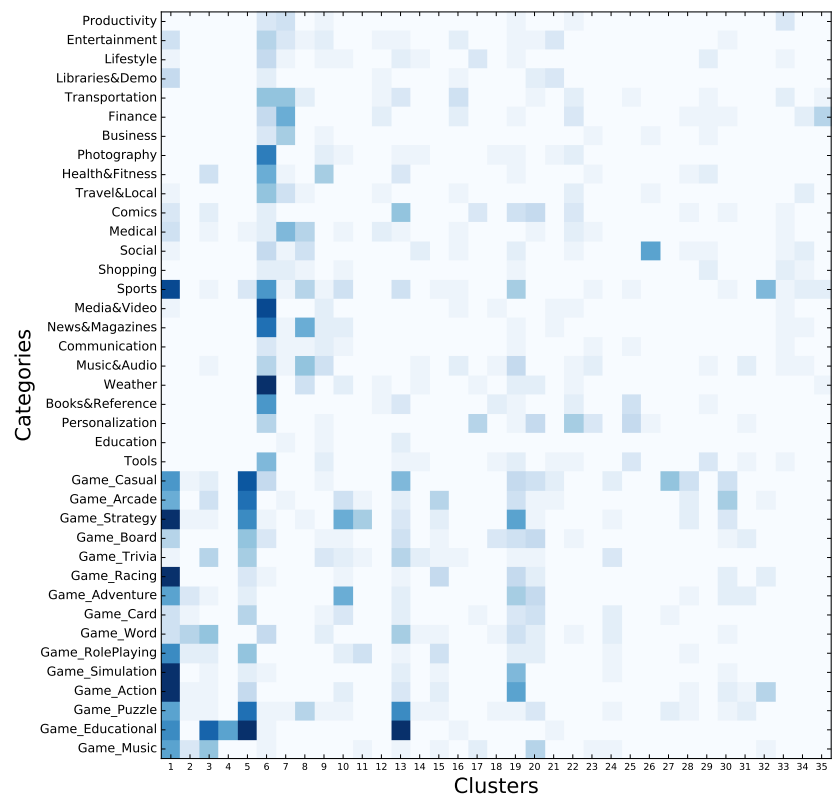

Fig. 8 Matrix for cluster result by category of apps from Google Play.

\subsubsection{Used sensor type}

According to the backward tracking analysis results, accelerometer is the most frequently used sensor. More than 70 percent of apps from AppChina and 66 percent of apps from Google Play use this sensor as indicated in Figure 9. This is mainly because some popular functions, such as Shake in WeChat (shake your phone, then you will find a random person who shakes his/her phone at the same time with you to chat with), need this sensor. All the widely used sensor related third-party libraries, such as Cocos2dx, Unity3D and OneKeyShare, require this sensor. Gyroscope is often used to display the 3D model of an object. Proximity sensor almost only appears in com.tencent.mm.sdk while gravity sensor almost only appears in Unity3D. The third-party libraries that provide map function, such 


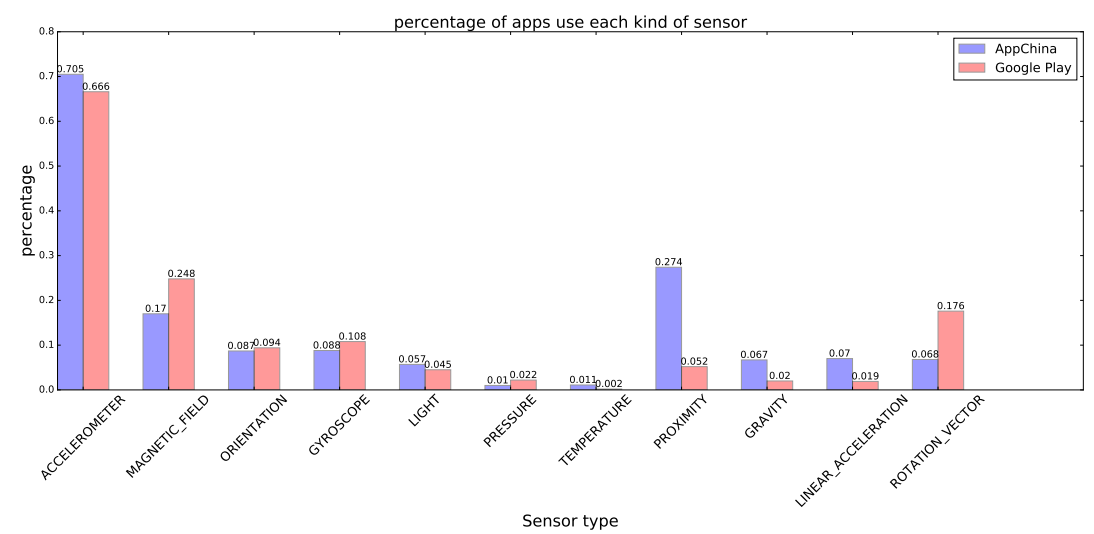

Fig. 9 Percentage of apps that use each type of sensor

as com.baidu.mapapi, will use the orientation sensor and magnetic field sensor. Some ad libraries also need magnetic field sensor. The apps that can distinguish QR Code may use the light sensor. Linear acceleration sensor is mainly used by Unity3D and rotation vector sensor is mainly used by Unity3D and Google ad. Motion sensors and position sensors are widely used, while environmental sensors are seldom used except the light sensor. This is because many smartphones do not have the hardware sensors to measure the temperature or humidity. Unity3D can access six types of sensors. However, madhouse, an ad library, registers eleven types of sensors for their ad display. Although attractive ads may need the data from embedded sensors, we do not think eleven types of sensors are necessary. We find 54 apps from AppChina and only one app from Google Play use this library. We summarize the detail about the used sensor types in the most used sensor related third-party libraries in Table 4. Apps from AppChina and most apps from Google Play only use the sensors showed in Figure 9, but several apps from Google Play also use some novel sensors. For example, WalkLogger pedometer from Health\&Fitness category used step detector sensor and step counter sensor. These two types of sensors were added in Android 4.4. WatchMaster-Watch Face from Personalization category used the heart rate sensor which was added in Android $4.4 \mathrm{~W}$.

\subsubsection{Discussion}

Our studies on AppChina and Google Play do not find any app that steals users' sensor data (The data read from the sensors which are not protected by Android permissions). Different from the methods of stealing users' location, contact or message, which often use Java reflection API to read data and send the data to the Internet, apps just use the sensor data in their local codes. However, since the embedded sensor can be directly used without requirement of permissions, some third-party libraries register all the sensors recklessly, no matter whether they really need. This not only leads to more power consumption, but also makes the sensors more easily be exploited by malicious apps. Though many apps contain the third-party libraries that can read sensor data, they do not functionally need 
Table 4 used sensor type in each third-party library

\begin{tabular}{|c|c|c|c|c|c|c|c|c|c|c|c|c|}
\hline 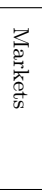 & Libraries & $\begin{array}{l}\vec{b} \\
0 \\
0 \\
0 \\
0 \\
0 \\
0 \\
0 \\
0 \\
0 \\
0\end{array}$ & 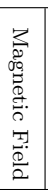 & 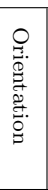 & 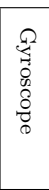 & 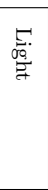 & 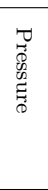 & 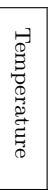 & 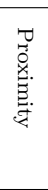 & 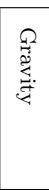 & 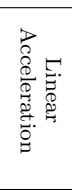 & 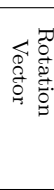 \\
\hline 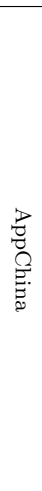 & $\begin{array}{l}\text { org.cocos2dx } \\
\text { com.unity3d.player } \\
\text { com.tencent.mm.sdk } \\
\text { cn.sharesdk.onekeyshare } \\
\text { com.umeng...UMShakeSensor } \\
\text { com.adsmogo.mriad } \\
\text { com.adchina.android.ads.views } \\
\text { com.madhouse.android.ads } \\
\text { com.millennialmedia.android } \\
\text { com.mobi.view } \\
\text { com.adcocoa } \\
\text { com.baidu.location } \\
\text { com.baidu.navi } \\
\text { com.amap.api.mapcore } \\
\text { com.google.zxing } \\
\text { com.badlogic.gdx } \\
\text { com.adobe.air } \\
\text { com.mobclix.android.sdk } \\
\text { com.trid.tridad }\end{array}$ & $\begin{array}{l}v \\
v \\
v \\
v \\
v \\
v \\
v \\
v \\
v \\
v \\
v \\
v \\
v \\
v \\
v\end{array}$ & $\begin{array}{l}v \\
v \\
v\end{array}$ & $v$ & $\begin{array}{l}v \\
v\end{array}$ & $\begin{array}{l}v \\
v\end{array}$ & $v$ & $\begin{array}{l}v \\
v\end{array}$ & $\begin{array}{l}v \\
v\end{array}$ & $v$ & $v$ & $v$ \\
\hline 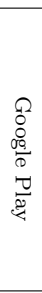 & $\begin{array}{l}\text { com.unity3d.player } \\
\text { co.google...overlay } \\
\text { org.cocos2dx.lib } \\
\text { com.adobe.air } \\
\text { com.tapjoy.mraid } \\
\text { com.ansca...CoronaSensorManager } \\
\text { com.mixpanel...FlipGesture } \\
\text { com.badlogic..edx } \\
\text { com.admarvel.android } \\
\text { com.facebook.ads } \\
\text { com.ideaworks3d...LoaderThread }\end{array}$ & $\begin{array}{l}v \\
v \\
v \\
v \\
v \\
v\end{array}$ & $\begin{array}{c}v \\
v \\
v \\
v\end{array}$ & $v$ & $v$ & $v$ & & & & $v$ & $v$ & $v$ \\
\hline
\end{tabular}

the sensor data. Hence, we suggest the third-party libraries put the sensor related codes in a standalone package, and let the developers chose to use it or not by themselves.

Runtime Performance We conducted the experiments on a quad-core machine with Inter Core $15 \mathrm{CPU}(3.10 \mathrm{GHz} \times 4)$ and $8 \mathrm{~GB}$ RAM, running Ubuntu 16.04 LTS. Most of the apps can be processed within 3 minutes. The experimental results indicate that SDFDroid is efficient to explore sensor usage behaviors of Android applications on a large scale.

Limitations Our method may not perform very well on the Apps with heavily smali code obfuscation. The Reflection API may also affect the analysis results. Another important factor affecting the analysis results is the use of Native methods. Some third-party development libraries write parts of their sensor related codes in the Native functions with the help of Native Development Toolkit(NDK). The Native functions are written by $\mathrm{C}++$ and cannot be disassembled to smali codes, hence they cannot be analyzed by SDFDroid. However, the state-of-art analysis tools like Flowdroid also meet these shortcomings. SDFDroid does not handle Android inter-component communication (ICC) and inter-process communication (IPC). But, since sensor data is used in highly real-time, we think it is seldom transported through ICC or IPC. SDFDroid uses the methods provided by Apktool to disassemble Android apps, hence we cannot analyze the apps that cannot be disassembled by Apktool. 


\section{Related Work}

The related work of this paper mainly falls into two aspects: string-based static analysis on Android apps and graph-based static program analysis of Android apps.

- String-based static analysis on Android apps. Many efforts have been made to perform static analysis on Android apps. Androgurad [11] is a toolset widely used in analyzing decompiled Android apps. It can be used to generate method invoke graph or detect malicious apps via signature matching. Androgurad cannot perform data tracking analysis . Another well-known static analysis tool for Android apps is FlowDroid [6] which is based on Soot [29]. It conducts precise static taint analysis on Android apps and generates the propagation path for sensitive data, but the analysis result relies on the predefined source and sink API of sensitive data. William Kliebe et al. [19] propose a tool combined FlowDroid and Epicc [27] to track both inter-component and intra-component data flow in a set of Android apps. Wei et al. [34] build a general framework called Amandroid for security analysis of Android apps. Li et al. [21] develop IccTA which improves the precision of the apps' inter-component analysis. However, this tool needs a very long time to analyze a single app. These tools need to generate a control flow graph for the whole app before they perform analysis, which cost much more time than SDFDroid that only concern sensor related codes. In other words, these tools are too heavyweight for the purposes of our study. The work which is most similar to ours is SAAF [17], a static Android analysis framework for Android apps. The difference between SDFDroid and SAAF is the that, SDFDroid has the ability to perform forward tracking analysis.

- Graph-based static program analysis of Android apps. Different from string-based static analysis that mainly focuses on the code strings, graph-based static analysis considers the function calls or data flow in the apps' program. Zhang et al. [38] propose ViewDroid, an approach to mobile app repackaging detection based on the apps' view graphs. Subgraph isomorphism algorithm is used in their work to measure the similarity between apps' view graphs. Gascon et al. [14] develop a method for Android malapps detection based on function call graphs. They employ an explicit mapping to map call graphs to a feature space, then train a support vector machine to distinguish malapps from benign apps. Zhang et al. [39] propose a novel approach to classify Android malapps via weighted contextual API dependency graph. They assign weights to different API nodes in the graph when measuring similarity by graph edit distance algorithm. That means critical APIs, such as API requiring permission check, have greater weights and more easily to influence the classification results. Chen et al. [10] develop a new technique called MassVet for vetting apps at a massive scale. Massvet uses two kinds of graphs. One is the app's view structure graph and the other one is the app's control flow graph. An algorithm called Centroids which is also proposed by Chen [9] is applied to calculate the app's $v$-cores and $m$-cores based on these two kinds of graphs. Then, they use these two kinds of cores to detect potential malicious apps fast and accurately at a massive scale. Elish et al. [12] propose a highly accurate classification approach for detecting malicious Android apps. Their method statically extracts a data 
dependence graph from a given app at first. And then they analyze how user inputs trigger sensitive API invocations based on this data dependence graph. The rate of sensitive API invocations which depended on user triggers is used to classify malicious Android apps. The difference between our work and the related work is that we use data flow graphs to do clustering while they use API call graphs for classification.

Although there exists related work on static analysis of Android apps, to the best of our knowledge, there is no work that has focused on thoroughly analyzing Android sensor usage behaviors. Different from our previous work on exploring the behaviors of sensor usage in Android apps from AppChina [23], we additionally analyze more than seven thousand apps from Google Play and present more detailed description of the system design, implementation, and experimental results, in order to better understand the Android sensor usage behaviors with data flow analysis based on all the apps from a complete Chinese Android market and popular apps from Google Play.

\section{Conclusion}

In this paper, we design and implement a tool called SDFDroid to analyze sensor usage behaviors in current Android apps. SDFDroid performs forward tracking analysis to generate an app's sensor data propagation graph and backward tracking analysis to find the app's used sensor types. Through extensive experiments on a widely-used Chinese App market, AppChina, and the official app market, Google Play, we find that Android apps often preform sensor related functions with the help of third-party libraries. Accelerometer is the most frequently used sensor. Though many apps only register one type of sensor, there are some apps registering eleven types of sensors. This indicates the emerging need for better regulating the sensor usage in Android apps. In the future work, we will extend the analysis to Native code, and generate more complete sensor data propagation graph to accurately characterize the app's sensor related functions. Besides, we will study on how to protect the sensors from the malicious attacks effectively with no influence on users' normal use.

\section{Acknowledgment}

The work reported in this paper is partially supported by the Fundamental Research funds for the central Universities of China (No. K15JB00190), Shanghai Key Laboratory of Integrated Administration Technologies for Information Security, the Ph.D. Programs Foundation of Ministry of Education of China (No. 20120009120010), the Scientific Research Foundation for the Returned Overseas Chinese Scholars, State Education Ministry (No. K14C300020), and in part by the 111 Project (B14005). 


\section{References}

1. Android. Android sensor overview. http://developer.android.com/guide/topics/ sensors/sensors_overview.html, 2015-03-28.

2. Android. Android sensor type. http://developer.android.com/reference/android/ hardware/Sensor.html, 2015-03-29.

3. Android. Dalvik bytecode. https://source.android.com/devices/tech/dalvik/ dalvik-bytecode.html, 2016.

4. Apktool. Apktool. http://ibotpeaches.github.io/Apktool/, 2015-05-20.

5. AppBrain. Google play stats. http://www.appbrain.com/stats/, 2016-10-28.

6. Steven Arzt, Siegfried Rasthofer, Christian Fritz, Eric Bodden, Alexandre Bartel, Jacques Klein, Yves Le Traon, Damien Octeau, and Patrick McDaniel. Flowdroid: Precise context, flow, field, object-sensitive and lifecycle-aware taint analysis for android apps. In Proceedings of the 35th ACM SIGPLAN Conference on Programming Language Design and Implementation, page 29. ACM, 2014.

7. Adam J Aviv, Benjamin Sapp, Matt Blaze, and Jonathan M Smith. Practicality of accelerometer side channels on smartphones. In ACSAC 2012, pages 41-50. ACM, 2012.

8. Liang Cai and Hao Chen. Touchlogger: inferring keystrokes on touch screen from smartphone motion. In Proceedings of the 6th USENIX conference on Hot topics in security, pages 9-9. USENIX Association, 2011.

9. Kai Chen, Peng Liu, and Yingjun Zhang. Achieving accuracy and scalability simultaneously in detecting application clones on android markets. In Proceedings of the 36th International Conference on Software Engineering, pages 175-186. ACM, 2014.

10. Kai Chen, Peng Wang, Yeonjoon Lee, XiaoFeng Wang, Nan Zhang, Heqing Huang, Wei Zou, and Peng Liu. Finding unknown malice in 10 seconds: Mass vetting for new threats at the google-play scale. In 24th USENIX Security Symposium (USENIX Security 15), pages 659-674, 2015.

11. Anthony Desnos. Androguard: Reverse engineering, malware and goodware analysis of android applications... and more (ninja!). http://code.google.com/p/androguard, 201303-26.

12. Karim O Elish, Xiaokui Shu, Danfeng Daphne Yao, Barbara G Ryder, and Xuxian Jiang. Profiling user-trigger dependence for android malware detection. Computers \& Security, 49:255-273, 2015.

13. Martin Ester, Hans-Peter Kriegel, Jörg Sander, and Xiaowei Xu. A density-based algorithm for discovering clusters in large spatial databases with noise. In $K d d$, volume 96 , pages 226-231, 1996.

14. Hugo Gascon, Fabian Yamaguchi, Daniel Arp, and Konrad Rieck. Structural detection of android malware using embedded call graphs. In Proceedings of the 2013 ACM workshop on Artificial intelligence and security, pages 45-54. ACM, 2013.

15. Gephi. The open graph viz platform. https://gephi.org, 2016.

16. Shohei Hido and Hisashi Kashima. A Linear-Time Graph Kernel. Ninth IEEE International Conference on Data Mining, 2009. ICDM '09., pages 179-188, December 2009.

17. Johannes Hoffmann, Martin Ussath, Thorsten Holz, and Michael Spreitzenbarth. Slicing droids: program slicing for smali code. In Proceedings of the 28th Annual ACM Symposium on Applied Computing, pages 1844-1851. ACM, 2013.

18. I.D.Corporation. Smartphone os market share, q2 2016. http://www.idc.com/prodserv/ smartphone-os-market-share.jsp, 2016-08.

19. William Klieber, Lori Flynn, Amar Bhosale, Limin Jia, and Lujo Bauer. Android taint flow analysis for app sets. In Proceedings of the 3rd ACM SIGPLAN International Workshop on the State of the Art in Java Program Analysis, pages 1-6. ACM, 2014.

20. Wei-Han Lee and Ruby B Lee. Multi-sensor authentication to improve smartphone security. In Conference on Information Systems Security and Privacy, 2015.

21. Li Li, Alexandre Bartel, Tegawendé François D Assise Bissyande, Jacques Klein, Yves Le Traon, Steven Arzt, Siegfried Rasthofer, Eric Bodden, Damien Octeau, and Patrick McDaniel. Iccta: detecting inter-component privacy leaks in android apps. In 2015 IEEE/ACM 37th IEEE International Conference on Software Engineering (ICSE 2015), 2015.

22. Chien-Cheng Lin, Deron Liang, Chin-Chun Chang, and Ching-Han Yang. A new nonintrusive authentication method based on the orientation sensor for smartphone users. In Software Security and Reliability (SERE), 2012 IEEE Sixth International Conference on, pages 245-252. IEEE, 2012. 
23. Xing Liu, Jiqiang Liu, and Wei Wang. Exploring sensor usage behaviors of android applications based on data flow analysis. In 34th IEEE International Performance Computing and Communications Conference, IPCCC 2015, Nanjing, China, December 14-16, 2015, pages 1-8, 2015.

24. Xing Liu, Sencun Zhu, Wei Wang, and Jiqiang Liu. Alde: Privacy risk analysis of analytics libraries in the android ecosystem. In 12th EAI International Conference on Security and Privacy in Communication Networks (SecureComm 2016), Guangzhou, China, October, 10-12, 2016, 2016.

25. Emiliano Miluzzo, Alexander Varshavsky, Suhrid Balakrishnan, and Romit Roy Choudhury. Tapprints: your finger taps have fingerprints. In Proceedings of the 10th international conference on Mobile systems, applications, and services, pages 323-336. ACM, 2012.

26. The Hacker News. Taplogger android trojan can determine tapped keys. http:// thehackernews . com/2012/04/taplogger-android-trojan-can-determine.html, 2012-0421.

27. Damien Octeau, Patrick McDaniel, Somesh Jha, Alexandre Bartel, Eric Bodden, Jacques Klein, and Yves Le Traon. Effective inter-component communication mapping in android with epicc: An essential step towards holistic security analysis. In Proceedings of the 22nd USENIX Security Symposium. Citeseer, 2013.

28. Emmanuel Owusu, Jun Han, Sauvik Das, Adrian Perrig, and Joy Zhang. Accessory: password inference using accelerometers on smartphones. In Proceedings of the Twelfth Workshop on Mobile Computing Systems \&3 Applications, page 9. ACM, 2012.

29. Soot. Soot. http://sable.github.io/soot/, 2015-12-09.

30. Raphael Spreitzer. Pin skimming: Exploiting the ambient-light sensor in mobile devices. In Proceedings of the 4th ACM Workshop on Security and Privacy in Smartphones 8 Mobile Devices, pages 51-62. ACM, 2014.

31. Dan Su, Wei Wang, Xing Wang, and Jiqiang Liu. Anomadroid: profiling android application behaviors for identifying unknown malapps. In 15th IEEE International Conference on Trust, Security and Privacy in Computing and Communications (IEEE TrustCom 2016), Tianjin, China, 23-26 August, 2016, 2016.

32. The Verge. Taplogger android app can read your password based on motion sensor data. http://www.theverge.com/2012/4/20/2963110/ taplogger-android-app-motion-sensor-data, 2012-04-20.

33. Wei Wang, Xing Wang, Dawei Feng, Jiqiang Liu, Zhen Han, and Xiangliang Zhang. Exploring permission-induced risk in android applications for malicious application detection. IEEE Trans. Information Forensics and Security, 9(11):1869-1882, 2014.

34. Fengguo Wei, Sankardas Roy, Xinming Ou, et al. Amandroid: A precise and general intercomponent data flow analysis framework for security vetting of android apps. In $C C S$ 2014, pages 1329-1341. ACM, 2014.

35. WIKI. Dbscan. http://en.wikipedia.org/wiki/DBSCAN, 2015-04-05.

36. Zhi Xu, Kun Bai, and Sencun Zhu. Taplogger: Inferring user inputs on smartphone touchscreens using on-board motion sensors. In Proceedings of the fifth ACM conference on Security and Privacy in Wireless and Mobile Networks, pages 113-124. ACM, 2012.

37. Zhiping Zeng, Anthony K. H. Tung, Jianyong Wang, Jianhua Feng, and Lizhu Zhou. Comparing Stars: On Approximating Graph Edit Distance. Proceedings of The Vldb Endowment, 2:25-36, 2009.

38. Fangfang Zhang, Heqing Huang, Sencun Zhu, Dinghao Wu, and Peng Liu. Viewdroid: Towards obfuscation-resilient mobile application repackaging detection. In Proceedings of the 7th ACM Conference on Security and Privacy in Wireless and Mobile Networks (WiSec 2014). Citeseer, 2014.

39. Mu Zhang, Yue Duan, Heng Yin, and Zhiruo Zhao. Semantics-aware android malware classification using weighted contextual api dependency graphs. In CCS 2014, pages 11051116. ACM, 2014.

40. Jiang Zhu, Pang Wu, Xiao Wang, and Joy Zhang. Sensec: Mobile security through passive sensing. In Computing, Networking and Communications (ICNC), 2013 International Conference on, pages 1128-1133. IEEE, 2013.

\section{Brief biography of the authors}

Xing Liu is a Ph.D. student in School of Computer and Information Technology, Beijing Jiaotong University, China. He received his B.S. degree from Beijing Jiaotong University in 2012. He visited the department of Computer Science and 
Engineering, The Pennsylvania State University, during October 2015-October 2016. His main research interests lie in mobile security and privacy.

Wei Wang is currently associate professor in the School of Computer and Information Technology, Beijing Jiaotong University, China. He earned his Ph.D. degree in control science and engineering from Xi'an Jiaotong University, in 2006. He was a postdoctoral researcher in University of Trento, Italy, during 2005-2006. He was a postdoctoral researcher in TELECOM Bretagne and in INRIA, France, during 2007-2008. He was a European ERCIM Fellow in Norwegian University of Science and Technology (NTNU), Norway, and in Interdisciplinary Centre for Security, Reliability and Trust (SnT), University of Luxembourg, during 2009-2011. He visited INRIA, ETH, NTNU, CNR, and New York University Polytechnic. He is young AE of Frontiers of Computer Science Journal. He has authored or co-authored over 40 peer-reviewed papers in various journals and international conferences. His main research interests include mobile, computer and network security.

Jiqiang Liu received his B.S. (1994) and Ph.D. (1999) degree from Beijing Normal University. He is currently a Professor at the School of Computer and Information Technology, Beijing Jiaotong University. He has published over 60 scientific papers in various journals and international conferences. His main research interests are trusted computing, cryptographic protocols, privacy preserving and network security.

Xiangliang Zhang is currently an Assistant Professor and directs the Machine Intelligence and kNowledge Engineering (MINE) Laboratory in the Division of Computer (http://mine.kaust.edu.sa), Electrical and Mathematical Sciences \& Engineering at King Abdullah University of Science and Technology (KAUST). She was an European ERCIM research fellow in the Department of Computer and Information Science, NTNU, Norway, from AprilAugust 2010. She earned her $\mathrm{PhD}$ degree in computer science with honors from INRIA-University Paris-Sud 11, France, in July 2010. She visited IBM T.J. Watson Research Center, Texas A\&M University, University Paris-Sud 11, Concordia University, Microsoft Research Asia and Rochester University. She regularly serves on the TPC for top-tier conferences like IJCAI 2017, AAAI 2017, WSDM 2017, KDD 2016 and ICDM 2016. She has authored or co-authored over 70 refereed papers in various journals and conferences. Her main research interests and experiences are in diverse areas of machine intelligence and knowledge engineering, such as complex system modeling, computer security and big data processing. 